\section{JTI}

JOURNAL OF

TRAUMA AND INJURY

\title{
Delayed Post-Traumatic Spinal Cord Infarction with Quadriplegia: A Case Report
}

\author{
Tae Hoon Kim, M.D.
}

Department of Emergency Medicine, Trauma Center, Wonkwang University Hospital, Iksan, Korea

Received: January 8, 2021

Revised: May 13, 2021

Accepted: May 26, 2021

\section{Correspondence to}

Tae Hoon Kim. M.D.

Department of Emergency Medicine, Trauma Center, Wonkwang University Hospital, 895 Muwang-ro, Iksan 54538 Korea

Tel: $+82-63-859-1650$

Fax: +82-63-859-1197

E-mail: doctor78em@gmail.com

ORCID: https://orcid.org/0000-00027683-7259

Traumatic spinal cord infarction is a rare condition that causes serious paralysis. The regulation of spinal cord blood flow in injured spinal cords remains unknown. Spinal cord infarction or ischemia has been reported after cardiovascular interventions, scoliosis correction, or profound hypotension. In this case, a 52-year-old man revisited the emergency center with motor and sensory abnormalities in all four extremities 56 hours after a motor vehicle collision. Despite the clinical presentation and imaging examination, there were no specific findings on the patient's first visit to the trauma center. Cervical spine computed tomography angiography showed a narrow vertebral artery, and diffusion-weighted imaging revealed spinal cord infarction from C3 to C5 with high signal intensity. It should be kept in mind that delayed-onset spinal cord infarction may occur in minor or major trauma patients as a result of head and neck injuries.

Keywords: Trauma; Spinal cord injuries; Quadriplegia

\section{INTRODUCTION}

Traumatic spinal cord infarction is a rare condition that encompasses various types of vertebral artery injuries, including occlusion, dissection, intimal tears, stenosis, and pseudo-aneurysm formation [1-4]. Delayed post-traumatic spinal cord infarction is a devastating complication that has been reported in children and adults after injuries without vertebral bone fractures [5-7]. Spinal cord ischemia has been reported to occur after aortic surgery, profound arterial hypotension, and intense exercise $[2,4,8]$, but traumatic spinal cord injuries after vehicle accidents have not been reported.

This case involved a 52-year-old male driver with severe complications, including progressive loss of strength in all four extremities and sensation below the shoulder,

This is an Open Access article distributed under the terms of the Creative Commons Attribution Non-Commercial License (http://creativecommons.org/licenses/by-nc/4.0/) which permits unrestricted noncommercial use, distribution, and reproduction in any medium, provided the original work is properly cited. 


\section{JTI}

56 hours after the vehicle collision. Specific aspects of this patient's clinical presentation, physical examination, computed tomography (CT), and magnetic resonance imaging (MRI) findings corresponded to a delayed traumatic spinal cord injury.

\section{CASE REPORT}

A 52-year-old man who was involved in a motor vehicle collision was transported to the trauma center by emergency medical services. His mental state was alert, but he exhibited loss of consciousness for about 1 minute. He complained of neck pain and a tingling sensation in his upper arms. A neurological examination revealed an extremity motor strength of Grade 4 and hyperesthesia in both upper arms, but a sensory examination revealed normal responses to temperature, light touch, pressure, and vibration. CT and MRI of the brain and cervical spine showed multiple degenerative changes that were mild at C3-C5 and within the normal limits for soft tissue, as well as no bony fractures (Fig. 1). The laboratory results showed no specific findings, and after 4 hours of observation, the patient's symptoms were relieved. The medical staff explained the necessity of close observation and monitoring symptoms in an inpatient setting despite symptom improvement, but the patient requested to be discharged.

The patient revisited the emergency center 56 hours after the accident. His vital signs were as follows: blood pressure, 176/85 mmHg; pulse rate, 64 beats per minute; respiratory rate, 20 breaths per minute; body tempera-
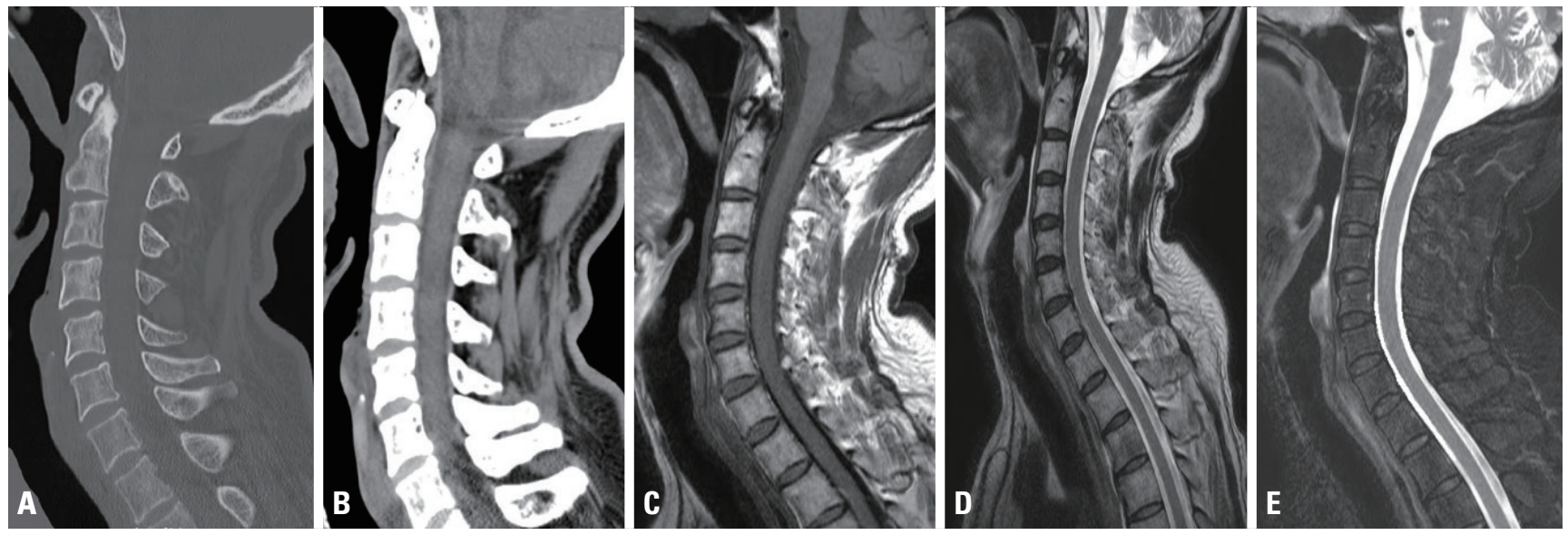

Fig. 1. No abnormalities are seen on (A) bone and (B) soft tissue imaging of the sagittal view on cervical spine computed tomography, (C) T1 turbo spin echo, (D) T2-weighted, and (E) T2-fat suppressed sagittal views of cervical-spine magnetic resonance imaging.
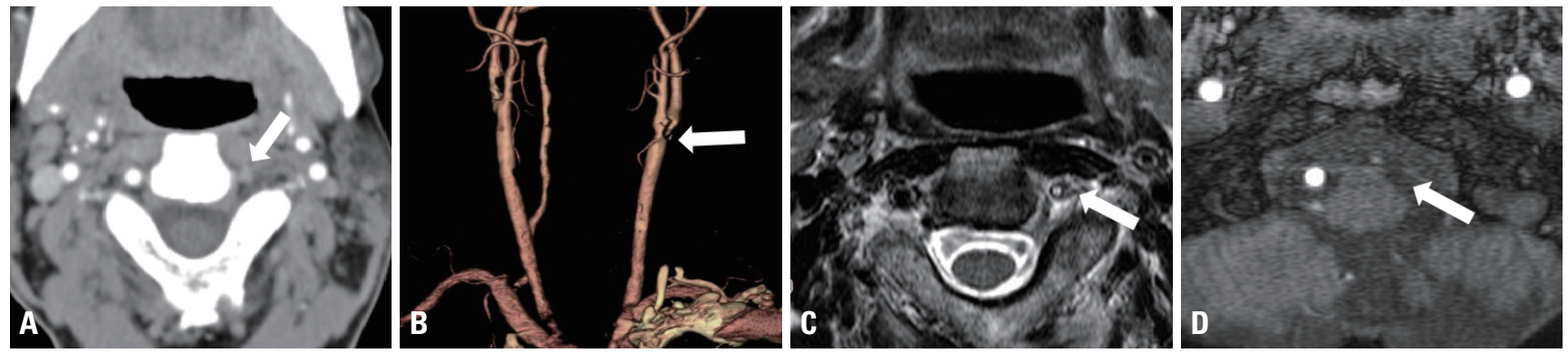

Fig. 2. Vertebral artery on the cervical spine level. (A) No filling contrast of the vertebral artery space (arrow), (B) severe stenosis of the proximal internal carotid artery on cervical spine computed tomography angiography (arrow), (C) lack of vertebral artery flow (arrow) on magnetic resonance imaging in a T2 axial view, and (D) absence of flow on cervical-spine magnetic resonance angiography (arrow). 
ture, $36.2^{\circ} \mathrm{C}$; and oxygen saturation, $99 \%$ on ambient air. He reported neck and shoulder pain, and the voluntary movement of both upper limbs suddenly became difficult.
After approximately 20 minutes, voluntary movement of the lower extremities became difficult. A neurological examination revealed quadriplegia, in which the motor
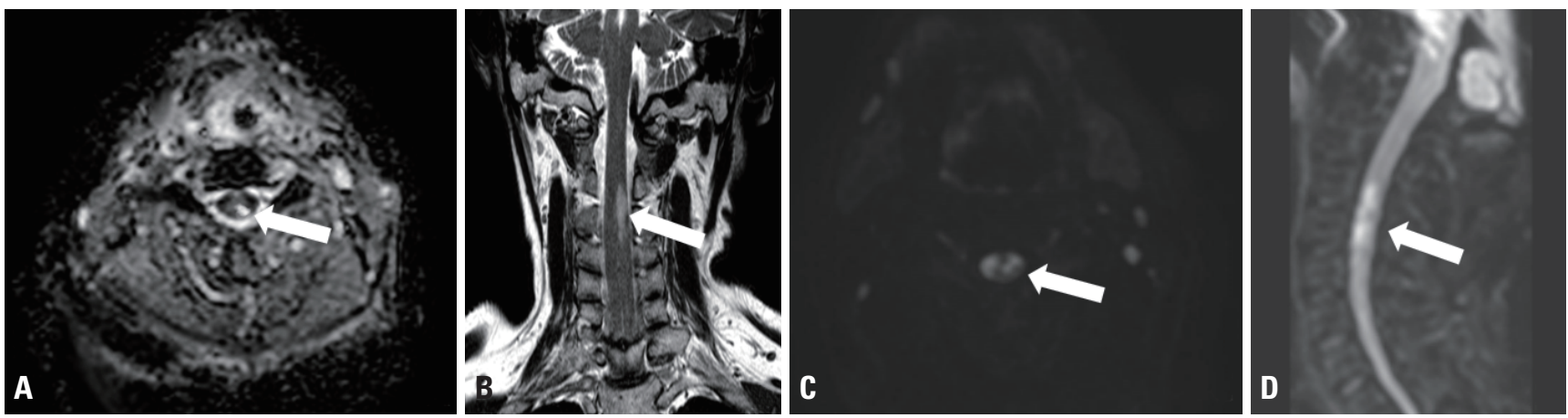

Fig. 3. Cervical-spine magnetic resonance imaging (MRI) 56 hours after the motor vehicle accident. Diffusion-weighted MRI with apparent diffusion coefficient (ADC) shows high signal intensity (arrows of A-D) on the (A) axial view, (B) coronal view, (C) diffusion-weighted imaging (DWI) b600 axial view, and (D) DWI b600 sagittal view of the cervical (C3-C5) spine.
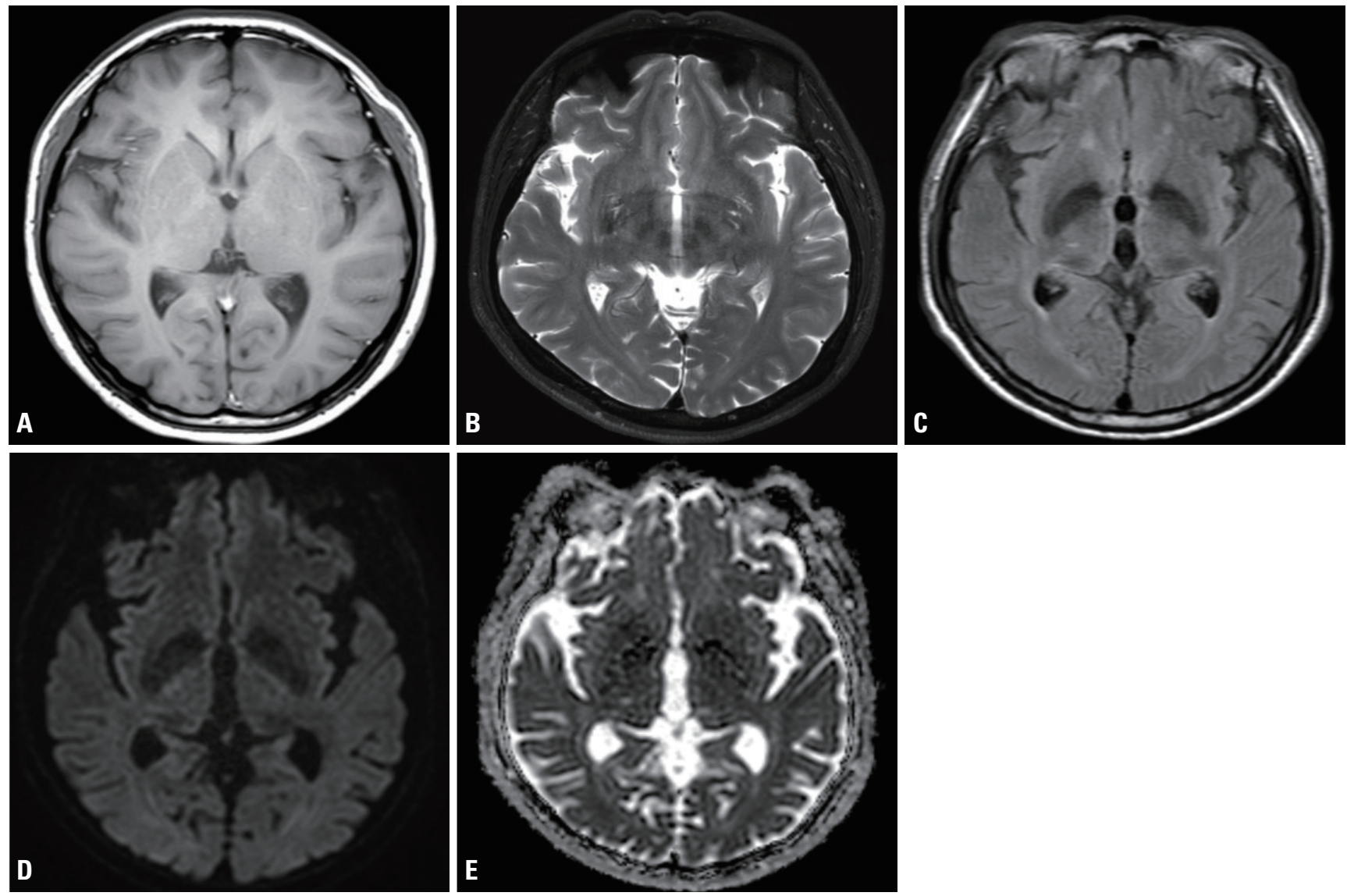

Fig. 4. Brain diffusion magnetic resonance imaging (MRI) after the patient was admitted to the intensive care unit on the next day. Diffusion-weighted MRI shows multiple small vessel infarctions with high signal intensity. (A) T1 axial view, (B) T2-diffusion-weighted imaging (DWI) axial view, (C) T2 fluid attenuated inversion recovery axial view, (D) DWI b2000 axial view, and (E) apparent diffusion coefficient (ADC) b2000 axial view in brain diffusion MRI. 
strength of the upper and lower extremities was classified as Grade 1, and a sensory examination revealed the absence of sensation of pain, temperature, and pressure bilaterally below level C4.

Cervical spine CT angiography and magnetic resonance angiography (MRA) suggested narrowing of the vertebral artery and the absence of flow. In addition, a proximal internal carotid artery showed severe stenosis (Fig. 2). The patient was suspected to have cervical spinal cord infarction and underwent diffusion-weighted imaging (DWI) of the cervical spine. MRI showed increased signal intensity at the vertebral level from C3 to C5 (Fig. 3). The patient immediately received heparinization and anticoagulation treatment, but spontaneous respiration deteriorated, and the oxygen saturation decreased after 8 hours. The patient's respiration gradually worsened, and his mental state changed to a deep drowsy state. Intubation was performed, and ventilator care was initiated to maintain respiratory control. After the patient was admitted to the intensive care unit, brain MRI with DWI was performed on the next day. The medical staff could not find any central nervous system problems in areas such as the cerebellum or brain stem that could cause the patient's loss of consciousness and respiratory failure (Fig. 4).

On the 14th day, the patient's mental state became alert and his vital signs were stable. Tracheostomy was performed, and spontaneous respiration was maintained with an oxygen supply and nebulizer treatment. On the 20th day, the patient was transferred to a rehabilitation hospital and continued to exhibit quadriplegia with sensory loss below the C4 level after 6 months.

\section{DISCUSSION}

Most cases of traumatic neck pain are associated with cervical sprains, strains, and contusion due to minor crashes or collisions. These symptoms of neck pain improve gradually, but delayed traumatic cervical spinal cord infarction is a devastating condition in rapid (fast) sequences that has not yet been described [2,6-10]. The differential diagnosis of acute progressive paraplegia after trauma cases includes spinal cord injury without radiographic abnormality (SCIWORA); this acronym was introduced by Pang and Wilberger, who used it to refer to clinical symptoms of traumatic myelopathy without findings of radiographic, CT, and MRI abnormalities $[11,12]$. In children, several cases of spinal cord infarction or ischemia after minor trauma have been reported $[1,6]$. A case series of children with spinal cord infarction without vertebral fractures concluded that hypotension and fibrocartilaginous embolism are principal etiological factors. However, several cases of spinal cord infarction in adults have suggested that injuries may be caused by avulsion of the perforating vessel, vasospasm of the artery of Adamkiewicz (radicular artery), or transient ischemia in areas of borderline perfusion resulting from flexion to extension of the spinal cord $[3,4,8]$. Ischemia of the spinal cord is believed to be the underlying mechanism of spinal cord injuries without radiographic abnormalities, but in the subacute phase, spinal cord ischemia typically manifests on MRI as focal cord swelling and "white pencil-like" high signal intensities on T2-weighted images $[6,7,9]$. Anatomic transection or instability of the spinal cord or large hematomas (greater than $1 / 2$ of the spinal cord diameter) have a poor prognosis and feature paresis or paralysis clinically $[7,9]$. As shown in this case report, the vascular territory is also an important factor in the anatomy of spinal cord infarction, which may manifest as abnormal findings of the vertebral artery on cervical spine CT angiography and MRA. The mechanism and timing of these vertebral circulation infarcts in spinal cord injury patients remain unclear $[4,7,11,12]$.

If a patient complains of temporary or rapidly recovering neck pain or back pain without any initial neurological problem or abnormal physical examination findings, consideration should be given to avoiding activities that may increase the risk of exacerbation or recurrence. Otherwise, the patient may experience paraplegia or death due to an unexpressed spinal cord injury. Therefore, physicians should be aware of the possibility of spinal cord injuries and utilize diagnostic tools such as CT angiography, MRA, and MRI with DWI for early-stage diagnosis, and the medical staff should explain the necessity of close observation and monitoring symptoms in an impatient setting for at least 48 hours even if symptoms improve. 


\section{CONFLICTS OF INTEREST}

No potential conflict of interest relevant to this article was reported.

\section{INFORMED CONSENT}

Informed consent was obtained from all individual participants included in this study.

\section{REFERENCES}

1. Lenn NJ. Spinal cord infarction due to minor trauma. Neurology 1977;27:999.

2. Mueller CA, Peters I, Podlogar M, Kovacs A, Urbach H, Schaller $\mathrm{K}$, et al. Vertebral artery injuries following cervical spine trauma: a prospective observational study. Eur Spine J 2011;20:22029.

3. Nance JR, Golomb MR. Ischemic spinal cord infarction in children without vertebral fracture. Pediatr Neurol 2007;36:209-16.

4. Torina PJ, Flanders AE, Carrino JA, Burns AS, Friedman DP, Harrop JS, et al. Incidence of vertebral artery thrombosis in cervical spine trauma: correlation with severity of spinal cord injury. AJNR Am J Neuroradiol 2005;26:2645-51.

5. Bartanusz V, Ziu M, Wood LE, Caron JL. Delayed post-traumatic spinal cord infarction in an adult after minor head and neck trauma: a case report. J Med Case Rep 2012;6:314.

6. Nagata K, Tanaka Y, Kanai H, Oshima Y. Acute complete paraplegia of 8-year-old girl caused by spinal cord infarction following minor trauma complicated with longitudinal signal change of spinal cord. Eur Spine J 2017;26:1432-5.

7. Taneichi H, Suda K, Kajino T, Kaneda K. Traumatically induced vertebral artery occlusion associated with cervical spine injuries: prospective study using magnetic resonance angiography. Spine (Phila Pa 1976) 2005;30:1955-62.

8. Compter A, van der Worp HB, Algra A, Kappelle LJ; Second Manifestations of ARTerial disease (SMART) Study Group. Prevalence and prognosis of asymptomatic vertebral artery origin stenosis in patients with clinically manifest arterial disease. Stroke 2011;42:2795-800

9. Iseli E, Cavigelli A, Dietz V, Curt A. Prognosis and recovery in ischaemic and traumatic spinal cord injury: clinical and electrophysiological evaluation. J Neurol Neurosurg Psychiatry 1999;67:567-71.

10. Nathanson A, Bird S, Dao L, Tam-Sing K. Competitive surfing injuries: a prospective study of surfing-related injuries among contest surfers. Am J Sports Med 2007;35:113-7.

11. Kothari P, Freeman B, Grevitt M, Kerslake R. Injury to the spinal cord without radiological abnormality (SCIWORA) in adults. J Bone Joint Surg Br 2000;82:1034-7.

12. Rozzelle CJ, Aarabi B, Dhall SS, Gelb DE, Hurlbert RJ, Ryken TC, et al. Spinal cord injury without radiographic abnormality (SCIWORA). Neurosurgery 2013;72(Suppl 2):227-33. 